\title{
A Load Identification Application Technology Based on Regularization Method and Finite Element Modified Model
}

\author{
Bingrong Miao $(\mathbb{D}$, Feng Zhou, Chuanying Jiang, Yaoxiang Luo, and Hui Chen
}

State Key Laboratory of Traction Power, Southwest Jiaotong University, Chengdu 610031, China

Correspondence should be addressed to Bingrong Miao; brmiao@home.swjtu.edu.cn

Received 22 April 2020; Revised 10 August 2020; Accepted 18 August 2020; Published 31 August 2020

Academic Editor: Giuseppe Petrone

Copyright (c) 2020 Bingrong Miao et al. This is an open access article distributed under the Creative Commons Attribution License, which permits unrestricted use, distribution, and reproduction in any medium, provided the original work is properly cited.

\begin{abstract}
An improved load identification technology of a beam based on different regularization methods and model modifying methods is presented in an attempt to minimize the estimation error at several periodic loads. A hybrid model is developed to simulate such ill-posed problem interactions under different noise levels. The finite element model is modified with the different optimization algorithms to obtain the equivalent constraint condition. Experimental verification is also carried out to obtain correct modes and frequencies by considered vibration response and different boundary conditions. The measured results demonstrate the good agreement with the identification results. The results are shown that the improved method not only has more adaptive range and higher identification accuracy but also has effective identification ability for loads under different noise levels.
\end{abstract}

\section{Introduction}

In recent decade years, extensive application of regularization method to engineering structures' health monitoring and inverse problems has been reported, as it has been recognized that using such an approach yields to improved quasi-static and dynamic load identification ill-posed problem characteristics [1]. The general concept for the solution of an ill-posed problem is to transform it into a wellposed problem by using additional information about the solution [2]. The methods, which transform the problem to the form of a well-posed problem, are regularization processes [3]. For these reasons, the regularization method had found its ways into aeronautical, civil, and mechanical engineering applications and started to become a standard method of solving inverse problem equation [4].

The literature on structural load identification is quite extensive and the research activities in this field have focused on a wide variety of applications. The identification problem was treated as an inverse problem to control certain objective functions. In other words, the structural load identification is reconstructed to achieve the optimization goal bounded by various constraints, among which is as consistent as possible with real loads under limited response data. Many scholars have proposed different identification methods for periodic and impact dynamic loads [2]. Thite and Thompson [4] discussed the relationship between Tikhonov regularization and the selection of regularization parameters by conventional crossvalidation methods and studied the iterative inversion technique of this method considering the influence of regularization matrix inversion. Liu and Han [5] proposed an inverse method which combines the interval analysis with regularization to stably identify the bounds of dynamic load acting on the uncertain structures. Liu et al. [6] found that most time-domain-based load identification methods based on state space have the disadvantages of large discrete error and long sampling time. The implicit Newmark- $\beta$ algorithm is transformed into a display algorithm to solve the load identification equation and uses Tikhonov regularization to solve the ill-conditioned problem. Sun et al. [7] combined a new improved regularization operator with L-curve method to overcome the illcondition of load reconstruction and obtained the stable and approximate solutions of inverse problems. Jia et al. [8] proposed a weighted regularization approach based on the proper orthogonal decomposition (POD), in which the regularization parameter was selected by the GCV method. Gao Wei et al. [9] proposed a new method for selecting 
regularized parameters. The method is based on the entropy function method of quadratic programming theory. $\mathrm{Nu}$ merical examples and experiments verify the effectiveness of the proposed method by identifying the sinusoidal loads acting on the cantilever beam. Prawin and Rao [10] use dynamic principal component analysis (DPCA) to propose an online identification load algorithm from the acceleration time history response measurement of moving windows. MAO et al. [11] proposed a time-domain force identification approach for the linear system, which is based on Markov parameter fine integration in the state space, and discussed the influence of the position of the measuring point and noise on the load identification result. In addition, Liu et al. [5] use Green pulse function or shape function to discretely represent the dynamic response relationship of the system, thus establishing a positive problem of load identification and combining different regularization methods to deal with the ill-posedness in the inverse problem of load identification to improve identification accuracy [12-15]. Miao et al. [16] also used the Green kernel function to establish the load identification equation and compared several regularization methods to solve the identification problem of sine and triangle loads.

However, fewer studies have considered the application of load identification to different regularization, and the modified model used different optimization method interaction problems [17-23]. Hence, it is the main objective of the current paper to solve load identification problem model using the modified finite element model. Through considering the boundary constraint and different optimization methods, the main vibration frequency of the measured mode is corrected.

\section{Theoretical Background and Mathematical Modelling}

2.1. Dynamics Equations Based on Duhamel Integrated Method. The structural dynamic equation of motion for a proportional damping system with multidegrees of freedom is described as

$$
\mathbf{M} \ddot{y}(t)+\mathbf{C} \dot{y}(t)+\mathbf{K y}(t)=\mathbf{F}(t),
$$

where $\mathbf{M}, \mathbf{C}$, and $\mathbf{K}$ are the total mass matrix, damping matrix, and stiffness matrix, respectively, $\mathbf{F}(t)$ is the load vector, and $\mathbf{y}(t), \dot{y}(t)$, and $\ddot{y}(t)$ are the displacement, velocity, and acceleration response vectors.

According to the characteristics of the Dirac function, the external excitation $f(t)$ of the system can be expressed as an integral form in which a plurality of pulse functions are superimposed. The equation can be described as

$$
f(t)=\int_{0}^{t} f(\tau) \delta(t-\tau) \mathrm{d} \tau
$$

It is assumed that the response of the system under the unit pulse load $f(\tau)$ is represented by the Green function $G(t)$ from the load action point to the response measurement point [3]. According to the principle of Dirac function superposition, the dynamic response caused by the external load of the system is described as

$$
y(t)=\int_{0}^{t} f(\tau) G(t-\tau) \mathrm{d} \tau .
$$

Considering the zero initial condition system, the convolution integral of equation (3) is transformed, where $\Delta(t)$ is represented as a discrete sampling time interval and $m$ is the number of sampling points. The equation can be discretized into a set of linear equations whose matrix form is described as

$$
\left\{\begin{array}{c}
y_{1} \\
y_{2} \\
\vdots \\
y_{m}
\end{array}\right\}=\left[\begin{array}{cccc}
G_{1} & & \\
G_{2} & G_{1} & \\
\vdots & \vdots & \ddots & \\
G_{m} & G_{m-1} & \cdots & G_{1}
\end{array}\right]\left\{\begin{array}{c}
f_{0} \\
f_{1} \\
\vdots \\
f_{m-1}
\end{array}\right\} \Delta t
$$

where $y_{i}, G_{i}$, and $f_{i}$ are the response of $t=i \Delta t$, the Green function, and the unknown external load. Furthermore, equation (4) can be simply described as

$$
\mathbf{Y}=\mathbf{G F} \text {. }
$$

Similarly, the identification problem of multiple load effects can be expressed as a matrix which is described as

$$
\left\{\begin{array}{c}
\mathrm{Y} 1 \\
\mathrm{Y} 2 \\
\vdots \\
\mathrm{Y} m
\end{array}\right\}=\left[\begin{array}{cccc}
\mathrm{G} 11 & \mathrm{G} 12 & \cdots & \mathrm{G} 1 n \\
\mathrm{G} 21 & \mathrm{G} 22 & \cdots & \mathrm{G} 2 n \\
\vdots & \vdots & \ddots & \vdots \\
\mathrm{G} m 1 & \mathrm{G} m 2 & \cdots & \mathrm{G}_{m n}
\end{array}\right]\left\{\begin{array}{c}
\mathrm{F} 1 \\
\mathrm{~F} 2 \\
\vdots \\
\mathrm{F} n
\end{array}\right\},
$$

where $m$ is the number of applied loads, $n$ is the number of response points, $\mathbf{Y}_{i}$ is the response, $\mathbf{F}_{i}$ is the column vector of the load, and $\mathbf{G}_{i j}$ is the Green Kernel Function between the load $\mathbf{F}_{j}$ and the response point $\mathbf{Y}_{i}$.

Since the actually measured response signal always contains noise, there is a small singular value in the Green kernel function matrix [17]. Equations (5) and (6) will become typical ill-conditioned equations. If not appropriately to directly invert the matrix $\mathbf{G}$, it is generally necessary to use an appropriate regularization technique to obtain an approximate solution to approximate the true result.

2.2. Regularization Technique. Regularization technology improves the ill-conditioning of load identification problem by modifying the singular value of system matrix and transforms the ill-conditioned problem into a steady-state problem [24-28]. Therefore, it provides an effective method for accurate load identification. The Tikhonov and TSVD regularization methods are used to be compared.

2.2.1. Tikhonov Regularization Technique. Tikhonov is derived from the variation method and is implemented by introducing stable functions [19]. For any $\alpha>0$, the quadratic function defined on the Hilbert space $\mathrm{X}$ is described as

$$
\Phi_{\alpha}(x)=\left\|A x-y^{\delta}\right\|+\alpha\|x\|^{2},
$$


where $\alpha$ is a regularization parameter and $y^{\delta}$ is represents $y$ value containing an error. The Tikhonov regularization method is to find the minimum point of the function $\Phi_{\alpha}(x)$ as an approximate solution to the equation.

It is known from the nature of the quadratic function that if the equation is satisfied, it can be obtained by equations (8) and (9):

$$
\begin{aligned}
x_{\alpha}^{\delta} & =\left(A^{*} A+\alpha I\right)^{-1} A^{*} y^{\delta}, \\
\mathbf{F}_{\alpha}^{\delta} & =\left(\mathbf{G}^{T} \mathbf{G}+\alpha \mathbf{I}\right)^{-1} \mathbf{G}^{T} \mathbf{Y}^{\delta} .
\end{aligned}
$$

2.2.2. TSVD Regularization Technique. Assuming that the generalized inverse of a linear equation exists, the solution can be described as

$$
\begin{aligned}
x^{+} & =A^{+} y \\
& =\sum_{i=1}^{\infty} \sigma_{i}^{-1}\left(y, v_{i}\right) u_{i},
\end{aligned}
$$

where $\sigma_{i}$ is the singular value of the compact operator $A$. The singular values are arranged in order from largest to smallest. $u_{i}$ and $v_{i}$ are the standard orthogonal bases of the subspace $N(A) \subset X$ and $R(A) \subset Y$. If the right end $y$ of the linear equation has a perturbation error $\delta_{y}$, such as $y^{\delta}=y+\delta_{y}$, the approximate solution of the linear equation is described as

$$
x^{\delta}=\sum_{i=1}^{\infty} \sigma_{i}^{-1}\left(y^{\delta}, v_{i}\right) u_{i} .
$$

And the error between $x^{\delta}$ and $x^{+}$is described as

$$
\begin{aligned}
\delta_{x} & =x^{\delta}-x^{+} \\
& =\sum_{i=1}^{\infty} \sigma_{i}^{-1}\left(\delta_{y}, v_{i}\right) u_{i} .
\end{aligned}
$$

It can be seen from the above equation that if the value of $\sigma_{i}$ is smaller, the influence of the disturbance error $\delta_{y}$ on the resulting error $\delta_{x}$ becomes larger. The truncated singular value decomposition regularization method is to cut off the right end of the equation for calculating the generalized solution. The solution only retains the first $k$ large singular values and filters the smaller singular values behind. Thus, the method can avoid the disturbance error being overamplified. The TSVD solution of the load identification equation (5) is described as

$$
f_{k}^{\delta}=\sum_{i=1}^{\infty} \sigma_{i}^{-1}\left(y^{\delta}, v_{i}\right) u_{i},
$$

where $f_{k}^{\delta}$ is the solution of the equation and $k$ is called the truncation step of the TSVD method and has the effect of correcting regularization parameters. $\left\{\sigma_{i}, u_{i}, v_{i}\right\}_{i=1}^{\infty}$ is a singular system for the matrix $\mathbf{G}$.

How to choose the optimal regularization parameter $k$ will directly affect the error and identification accuracy between the identification load and the real load. The GCV criterion is selected as the parameter selection method for TSVD regularization.

2.2.3. Selection of Regularization Parameters. It can be known from (9) and (13) that it is essential to select the optimal regularization parameters when the regularization technique solves the load identification equation. The Tikhonov regularization method uses the L-curve criterion for regularization parameter selection, while the TSVD regularization method is combined with the GCV criterion for research. The size of the regularization parameter will directly affect the error between the identification load and the real load. Here is a brief description of two regularization parameter selection strategies.

(1) L-Curve Criterion. In the optimization problem of Tikhonov regularization, the norm $\left\|f_{\alpha}^{\delta}\right\|_{2}$ of the regularization and the residual norm $\left\|y^{\delta}-G f_{\alpha}^{\delta}\right\|_{2}$ are functions of the regularization parameter. Taking these two variables as the horizontal and vertical coordinates, and the resulting curve is as follows. In the general coordinate system, the curve will appear as a distinct $\mathrm{L}$ shape. The corner of $\mathrm{L}$ curve is the norm between the regularization and the residual value to obtain the optimal equilibrium position. The corresponding parameter of this corner point is the optimal regularization parameter. In order to reasonably determine the position of the corner of the curve, the maximum curvature point of the curve at the scale is generally selected. Assign the parameter $\rho=\log \left\|y^{\delta}-H f_{\alpha}^{\delta}\right\|_{2}, \quad \theta=\log \left\|f_{\alpha}^{\delta}\right\|_{2} ;$ then, the curvature equation of the $\mathrm{L}$ curve is described as

$$
L(\alpha)=\frac{\left|\rho^{\prime} \theta^{\prime \prime}-\rho^{\prime} \theta^{\prime}\right|}{\left[\left(\rho^{\prime}\right)^{2}+\left(\theta^{\prime}\right)^{2}\right]^{3 / 2}},
$$

where $\rho^{\prime}, \theta^{\prime}$, and $\theta^{\prime \prime}$ are the first and second derivatives of the regularization parameter, respectively. The optimal regularization parameters determined by the L-curve criterion should be satisfy the following condition:

$$
L\left(\alpha_{o p}\right)=\max _{\alpha>0} L(\alpha) .
$$

(2) GCV Curve Criterion. The generalized crossvalidation (GCV) curve criterion is based on the PRESS criterion in statistical theory. And it is more robust than the PRESS criterion. The basic idea of the GCV method is that reasonable regularization parameters can effectively predict any new unknown load. The equation defining the GCV function is described as

$$
G C V(\alpha)=\frac{\left\|\left(\mathbf{I}-\mathbf{A}(\alpha)^{\mathbf{y} \delta}\right)\right\|_{2}^{2}}{\left.{ }_{\operatorname{Tr}}(\mathbf{I}-\mathbf{A}(\alpha))\right)^{2}},
$$

where $\mathbf{A}(\alpha)=\mathbf{G}\left({ }^{\mathbf{G} T} \mathbf{G}+\alpha \mathbf{I}\right)^{-1 \mathbf{G T}}$ and $\mathbf{T}_{\mathbf{r}}$ represents the trace of the matrix. And the equation satisfies the condition 
$\alpha\left({ }^{\mathbf{G} T} \mathbf{G}+\alpha \mathbf{I}\right)^{-1}=\mathbf{I}-\mathbf{G}\left({ }^{\mathbf{G} T} \mathbf{G}+\alpha \mathbf{I}\right)^{-1 \mathbf{G T}}$; then, equation (16) can be simplified to the following equation:

$$
G C V(\alpha)=\frac{\left\|\left({ }^{\mathbf{G} T} \mathbf{G}+\alpha \mathbf{I}\right)^{-1 \mathbf{y} \delta}\right\|_{2}}{\operatorname{Tr}\left(\left({ }^{\mathbf{G} T} \mathbf{G}+\alpha \mathbf{I}\right)^{-1}\right)}
$$

The criteria for selecting the optimal regularization parameters according to the GCV method are described as

$$
\operatorname{GCV}\left(\alpha_{\mathrm{op}}\right)=\min _{\alpha>0} G C V(\alpha) \text {. }
$$

In solving this minimization problem, the numerator term of the GCV function in equation (17) is actually the residual norm of the regularization solution. This item is easier to obtain, but when the matrix dimension is large, the function of the denominator matrix trace of the function is very large.

\section{The Improved Load Identification Algorithm}

The load identification technology for periodic loads proposed in this paper is an inverse problem analysis method based on finite vibration response data, which mainly considers the problem of large fluctuations in the identification results due to differences in model accuracy and boundary conditions [29-35]. In order to avoid the uncertainty of the boundary conditions and structural parameters of the actual structural model from affecting the recognition accuracy, the recognition method proposed in this paper takes into account the model modification, using the results of the calculated and experimental modalities and several optimization algorithms to modify the limited boundary conditions and structural parameters of the meta model. Based on the abovementioned theoretical formula, load identification is carried out through vibration response. At the same time, the recognition accuracy of several regularization methods after model modification is compared in the algorithm. The specific flow chart is shown in Figure 1.

The improved algorithm of load identification is listed as follows:

Step 1: establish numerical and experimental models of Euler-Bernoulli beam structure according to actual conditions.

Step 2: use experimental modal and computational modal analysis methods to obtain the modal and mode shapes of the beam structure under different boundary conditions.

Step 3: determine the type and number of sensors based on the condition number of the system matrix and the criteria for obtaining the vibration response accurately. The selection requirements of sensor location need to improve the noise immunity, signal-to-noise ratio, and reliability of the data as much as possible.

Step 4: according to the analysis results of the calculated mode and the experimental mode, an optimization method is selected to modify the boundary conditions and structural parameters of the finite element model to ensure that the relative error between the finite element mode and the experimental mode is minimized.

Step 5: after confirming that the model correction result satisfies the conditions, apply the type, size, and position of the periodic load on the numerical model and the experimental model.

Step 6: carry out on-site verification test according to the measured load to obtain the measured vibration response data.

Step 7: use Picard's condition to determine the wellposedness of the inverse problem.

Step 8: perform load identification simulation according to the finite element simulation model, and calculate the vibration response.

Step 9: obtain the measured data of the vibration response according to the experimental model.

Step 10: according to Green's function and different regularization algorithms, considering different degrees of noise, use vibration response data to calculate the kernel function matrix.

Step 11: determine the optimal regularization parameter (GCV curve and L-curve) according to the regularization parameter selection criterion to reduce the influence of noise and boundary conditions.

Step 12: if it is an ill-posed problem, determine the appropriate regularization technique to solve the load identification matrix equation.

Step 13: compare and verify the simulation results and experimental results and evaluate the effectiveness of the recognition algorithm.

\section{Numerical Example}

4.1. Simulation Model for Load Identification. As a typical force structure, the cantilever beam structure can be used to identify the dynamic load, which is of great significance for both theoretical research and engineering practice. Therefore, the cantilever beam structure is selected as a numerical simulation model for periodic dynamic load identification, as shown in Figure 2. The cantilever beam has a structural size of $0.8 \mathrm{~m} \times 0.06 \mathrm{~m} \times 0.004 \mathrm{~m}$, and the elastic modulus of the steel material is $2.1 \times 10^{11} \mathrm{~Pa}$, the density is $7.8 \times 10^{3} \mathrm{~kg} / \mathrm{m}^{3}$, and Poisson's ratio is 0.3 . It is assumed here that the structural damping of the cantilever beam is proportional damping.

The vibration data is obtained to simulate the measured response through adding white Gaussian noise to the simulation model. The response with noise is described as

$$
\mathbf{y}^{\delta}=\mathbf{y}^{c}+l^{\delta} \cdot \operatorname{std}\left(\mathbf{y}^{c}\right) \cdot \operatorname{randn},
$$

where $y^{c}$ is the response calculated by the simulation, which can be displacement, velocity, and acceleration, $\operatorname{std}\left(y^{c}\right)$ is the standard deviation of the calculated response, $l^{\delta}$ is a percentage representing the noise level, And randn represents a white noise random number of the same length as $\mathrm{y}^{\mathrm{c}}$, where the mean is 0 and the variance is 1 . 


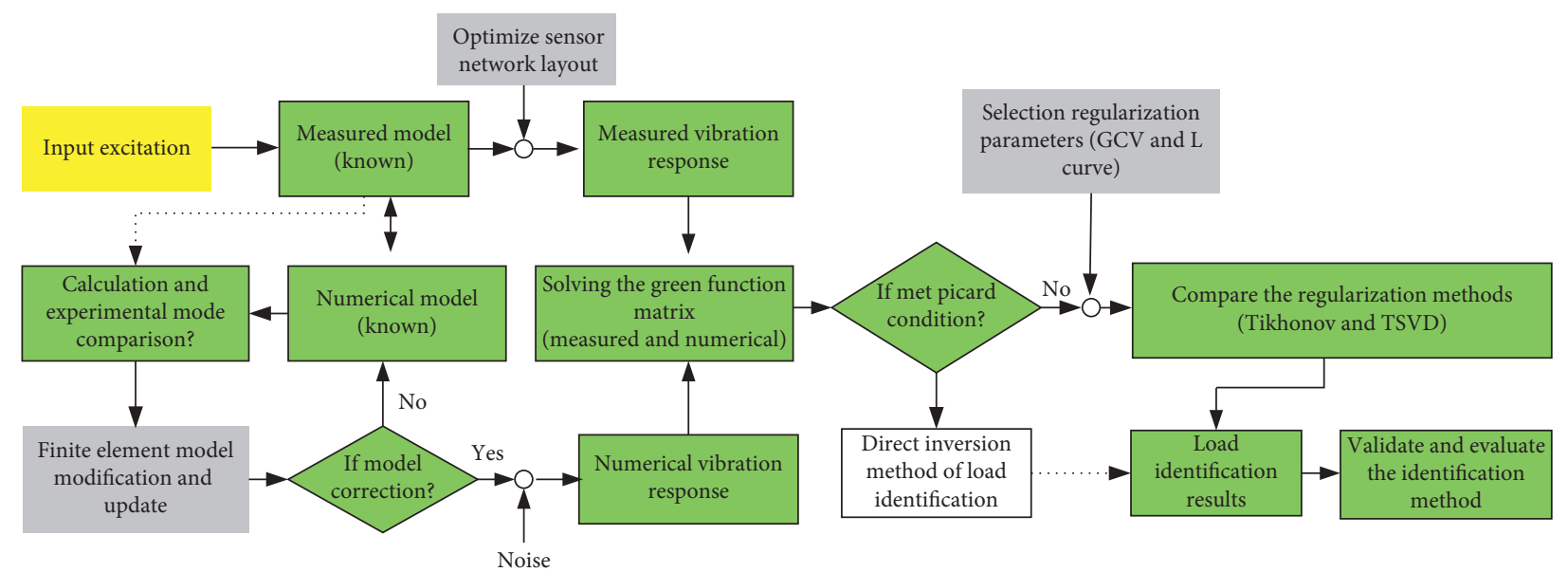

FIGURE 1: Load identification improved algorithm based on modified finite element model.

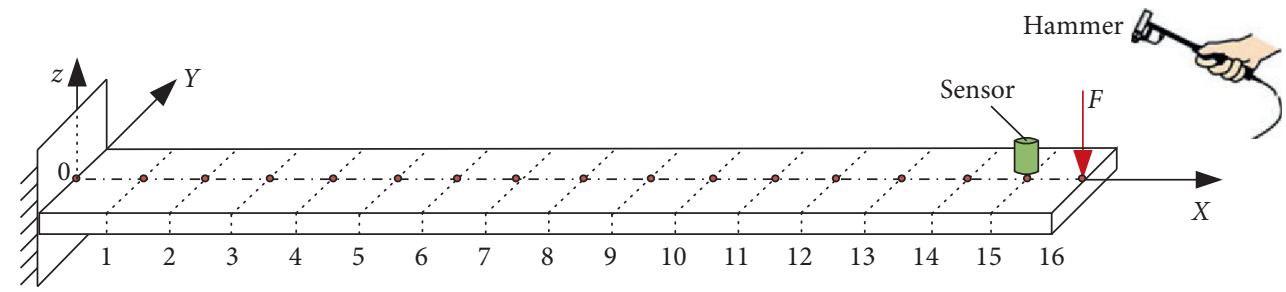

Figure 2: Numerical simulation model of the cantilever beam.

In order to evaluate the degree of conformity between the identification load and the real load, a relative error function is introduced, which is described as

$$
R E(i)=\left|\frac{F_{\text {Real }}(i)-F_{\text {ident }}(i)}{F_{\text {ident }}(i)}\right| \times 100 \% \text {, }
$$

where $i=1,2, \ldots, n$, and $n$ is the number of sampling points.

In addition, in order to describe the effectiveness of the identification method, two indicators of total relative error (RE) and correlation coefficient (CC) are required. The equation is described by equations (21) and (22):

$$
\begin{aligned}
& R E=\frac{\left\|\mathbf{f}^{\alpha, \delta}-\mathbf{f}\right\|}{\|\mathbf{f}\|} \times 100 \%, \\
& C C=\frac{\sum_{i=1}^{m}\left[f_{i}^{\alpha, \delta}-\mathrm{E}\left(\mathbf{f}^{\alpha, \delta}\right)\right]\left[f_{i}-E(\mathbf{f})\right]}{\left\|\mathbf{f}^{\alpha, \delta}-E\left(\mathbf{f}^{\alpha, \delta}\right)\right\|}\|\mathbf{f}-E(\mathbf{f})\|,
\end{aligned}
$$

where $E(\mathbf{f})$ and $E\left(\mathbf{f}^{\alpha, \delta}\right)$ are the average of the actual load and the identified load, respectively.

A unit pulse load is applied to the above excitation point (corresponding to the node 16 of the simulation model), and the Green pulse kernel function response of the excitation point to the response point (node 15) is obtained by finite element calculation, and a corresponding kernel function matrix $G$ is established [36, 37]. At 5\% white noise level, the TSVD regularization identification results are compared with the traditional Tikhonov regularization method. The identification comparison results of sinusoidal loads at 5\% noise level is shown in Figure 3.

The relative errors of these two regularization methods in identifying sinusoidal loads in the whole time period are obtained. The relative errors results are described in Figure 4.

It can be seen that both the TSVD regularization and the Tikhonov regularization can identify the added sinusoidal load. Obviously, the load identified by the Tikhonov method is fluctuated greatly, while the TSVD method is closer to the true load value. The error of TSVD regularization identification is lower than the Tikhonov regularization at most time points, and the relative error value can be guaranteed to be around $10 \%$ or less. The relative error of Tikhonov regularization is basically above $15 \%$, and the overall relative error of identification is $21.36 \%$. It shows that TSVD regularization identification can more accurately and stably identify the applied sinusoidal load compared to Tikhonov. In addition, the reason for the higher individual peak point identification error is due to the smoothing effect of the TSVD method on the identified load during the regularization process.

In order to further analyze the identification effect of TSVD regularization, Table 1 shows the overall relative error and correlation coefficient values identified by the TSVD method and the Tikhonov method under different noise levels, and the TSVD regularization is selected at $10 \%, 20 \%$, and $30 \%$ noise levels.

It can be seen from Table 1 that, as the noise level increases, the overall relative error of the TSVD and Tikhonov regularization methods increases, and the correlation 


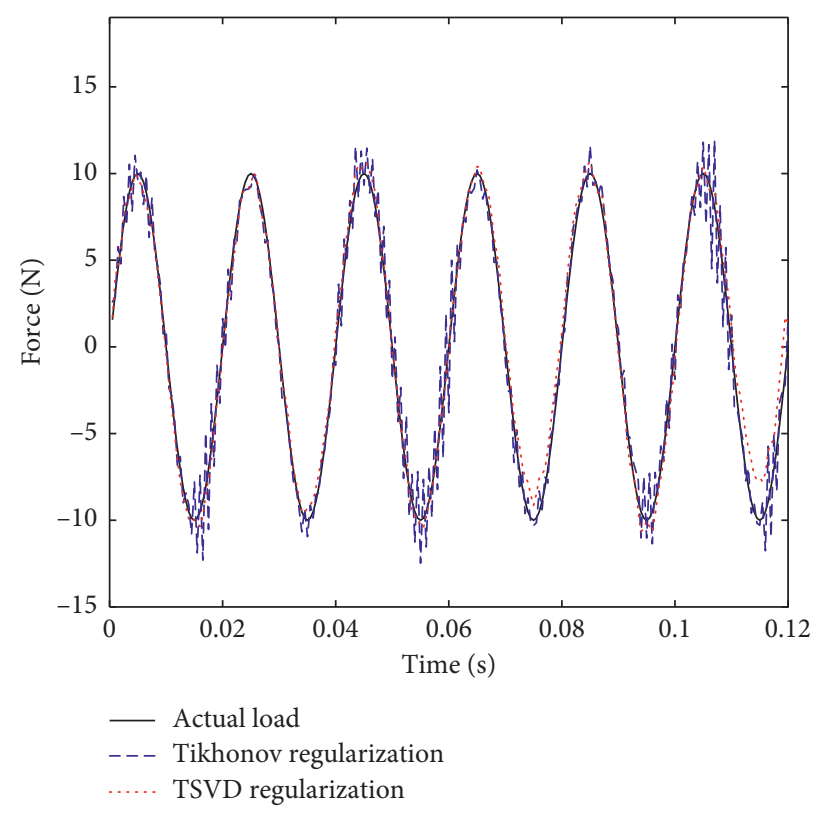

Figure 3: Identification results of sinusoidal loads at 5\% noise level.

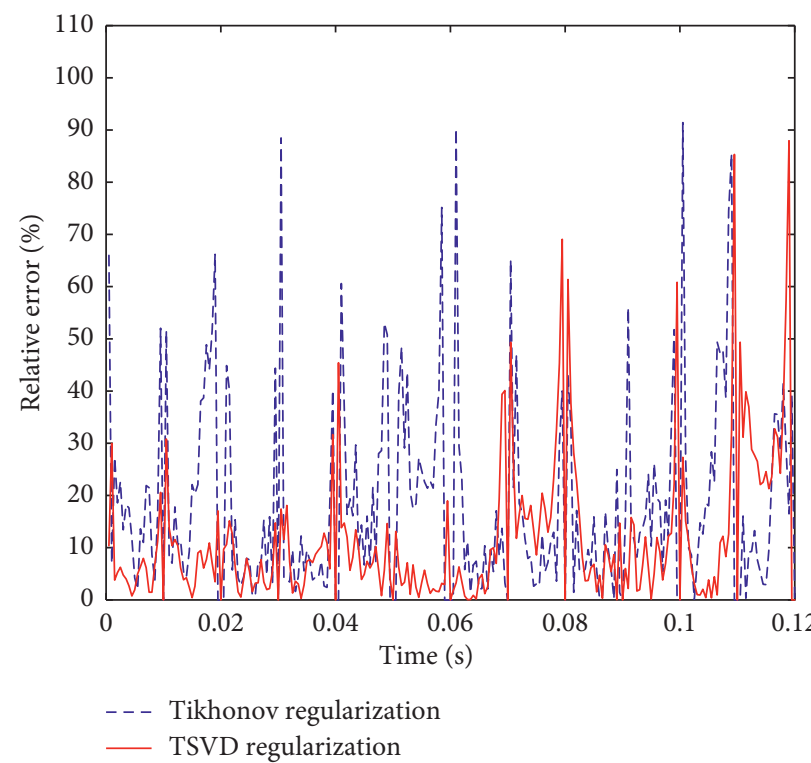

Figure 4: Relative error of sinusoidal load identification at 5\% noise level.

TABLE 1: Identification error at different noise levels.

\begin{tabular}{lcccc}
\hline \multirow{2}{*}{ Noise level } & \multicolumn{2}{c}{ Overall relative error RE (\%) } & \multicolumn{2}{c}{ Correlation coefficient CC } \\
& TSVD method & Tikhonov method & TSVD method & 0.9921 \\
Tikhonov method \\
\hline 5 & 12.54 & 21.36 & 0.9908 & 0.9777 \\
10 & 13.55 & 31.08 & 0.9868 & 0.9543 \\
20 & 16.17 & 47.0 & 0.9811 & 0.9033 \\
30 & 19.37 & 63.71 & 0.9735 & 0.8402 \\
40 & 22.95 & 80.72 & 0.9635 & 0.7732 \\
50 & 26.95 & 97.97 & & 0.7077 \\
\hline
\end{tabular}


coefficient decreases. However, the relative error identified by the Tikhonov method increases by more than $10 \%$, and the correlation coefficient has dropped to 0.77 when the noise level is $40 \%$. The error identified by the TSVD method is only increased by about $3 \%$ each time, and the correlation coefficient can be guaranteed to be above 0.96 . This shows that the TSVD regularization has a better suppression of noise, and the applied load can be more accurately recognized even at a higher noise level (20\%). The results are shown in Figure 5.

A periodic mixed sinusoidal load, a triangular load, and a square wave load are applied to the free end of the beam (node 16), respectively, and 5\% of the white noise is added to the acceleration response on node 15 , using the response and TSVD. Regularization is performed for load identification, and the results obtained are shown in Figures 6-8. For the triangular and mixed sinusoidal loads, the respective identification results at five time points are shown in Table 2.

Tables 2 and 3 show that the Tikhonov identification load error is basically higher than TSVD at these five time points, whether it is sinusoidal load or triangular load, and the error of some points is even more than $20 \%$, and the relative error of TSVD is $10 \%$. At the same time, the correlation coefficient of the TSVD regularization identification load is above 0.99 , while the Tikhonov is below 0.99 . Therefore, from the above chart, the feasibility of the TSVD regularization method to identify the periodic load and the superiority with respect to Tikhonov are explained in the acceleration response as the load identification input.

The results in the figures and tables show that the TSVD regularization method is more efficient than the Tikhonov regularization method. Furthermore, the fluctuation and relative error of the identified loads are smaller. In addition, due to the transient abrupt change of the square wave load, the TSVD method recognizes that when identifying the type of load, the identification error is large at some points in time, and the overall identification error is higher than the other two types of loads.

\subsection{Experiment Validation}

4.2.1. Establishment of Experimental Model. From the previous analysis results, it can be seen that the uncorrected finite element model still has a certain error in the load identification. As the modal order increases, the error will be greater. It is necessary to establish a modified finite element model to improve the recognition effect. Therefore, this article established a detailed experiment validation plan. The experimental object is a cantilever steel beam with the same material and size as the simulation model. This article mainly analyzes the structural modal test data in the DH5923 software. The sampling frequency is $2 \mathrm{kHz}$. And the equivalent constraint boundary conditions can be simplified by linear springs and torsion springs. The linear spring stiffness is set to $1 \times 10^{5} \mathrm{~N} / \mathrm{m}$, and the torsion spring stiffness is set to $1 \times 10^{6} \mathrm{~N} / \mathrm{m}$. The experimental scene is as shown in Figure 9.

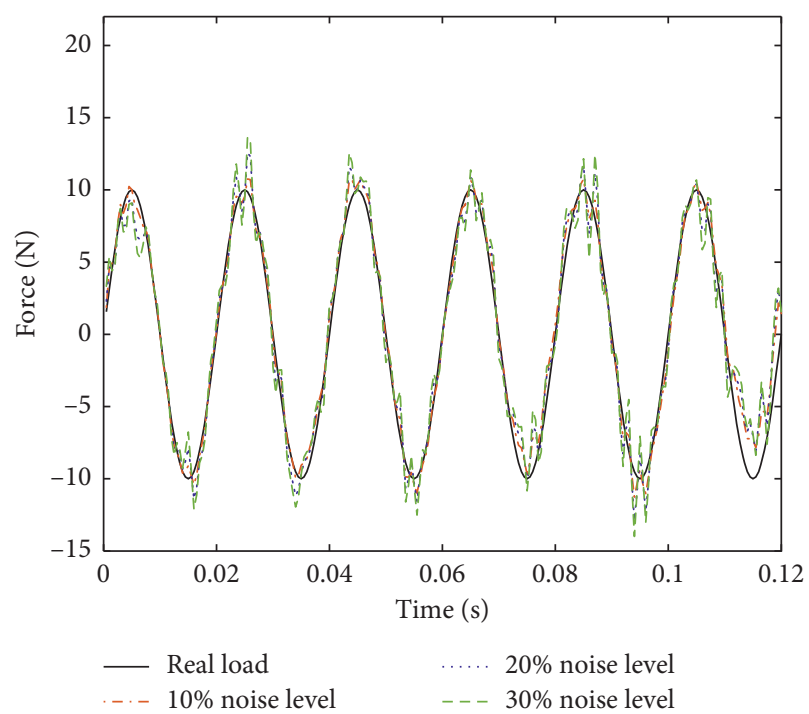

FIGURE 5: Identification results of TSVD under 10\%, 20\%, and 30\% noise levels.

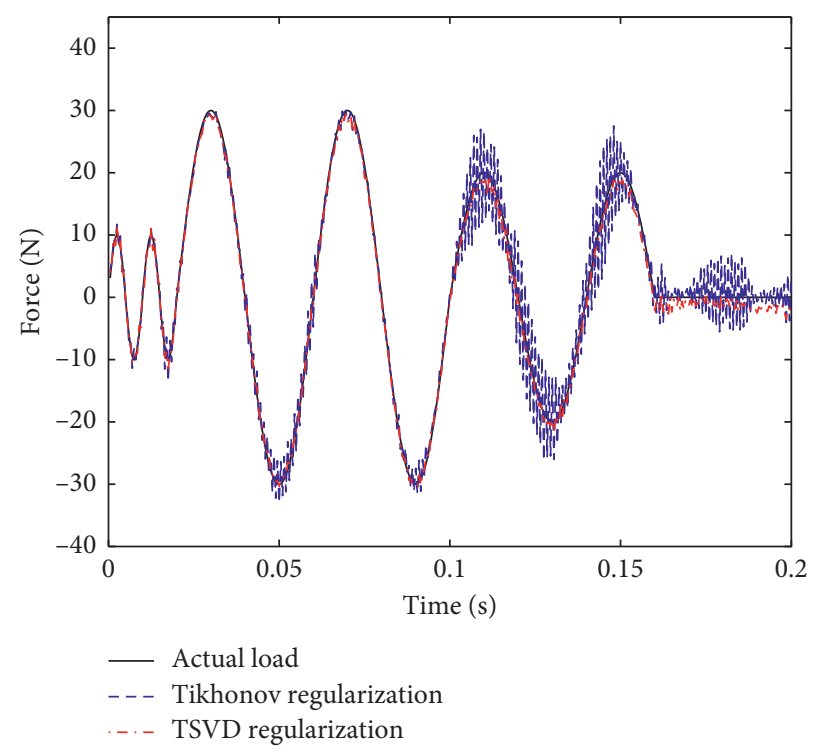

FIGURE 6: Identification results of mixed sinusoidal loads under 5\% noise.

The modal analysis of the finite element model is carried out, and the comparison of finite element modal and experimental modal frequencies of the beam structure is listed in Table 4.

From the table, it is found that the relative errors of the first and second order modal frequencies obtained by experimental measurements and finite element analysis are small, while the relative errors of other orders are greater than $5 \%$. This shows that the modal frequencies calculated by the finite element method are quite different from the measured frequencies, and the finite element model needs to be revised. Therefore, the first and second order modal frequency is chosen as the modification target of finite element model updating. 


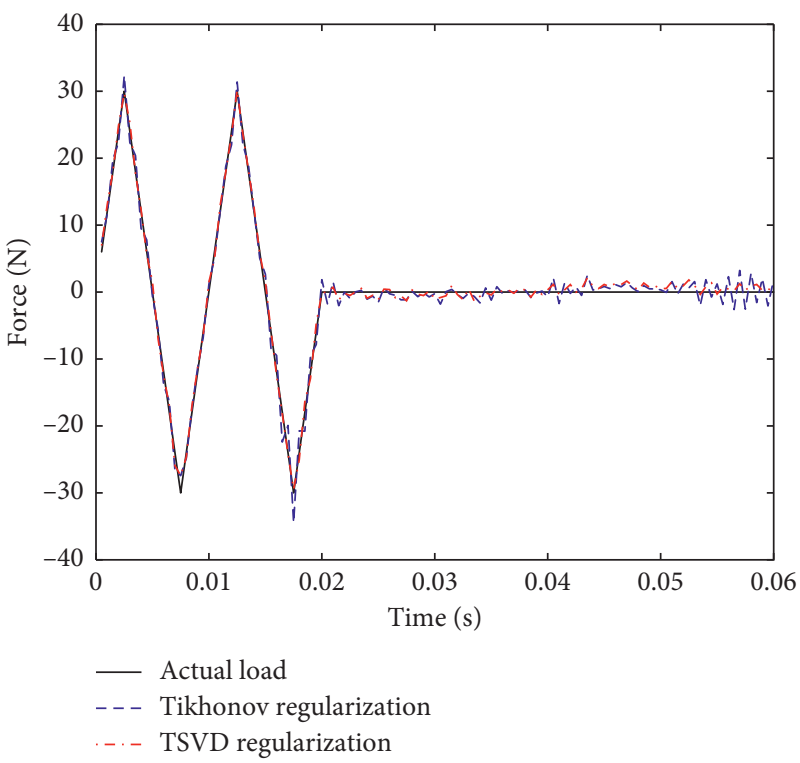

Figure 7: Identification results of triangular loads under 5\% noise.

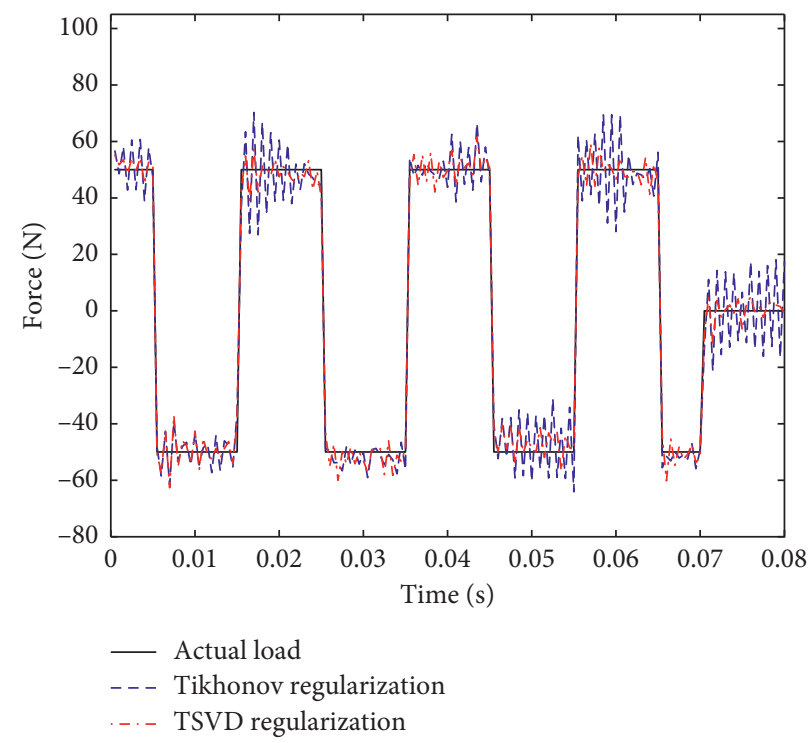

FIGURE 8: Identification results of wave loads under 5\% noise.

TABLE 2: Load identification results at five time points under $5 \%$ noise.

\begin{tabular}{|c|c|c|c|c|c|c|}
\hline \multirow{2}{*}{ Type } & \multirow{2}{*}{ Time (s) } & \multirow{2}{*}{ Actual load (N) } & \multicolumn{2}{|c|}{ Tikhonov regularization } & \multicolumn{2}{|c|}{ TSVD regularization } \\
\hline & & & Identified load $(\mathrm{N})$ & Error (\%) & Identified load $(\mathrm{N})$ & Error (\%) \\
\hline Sine & 0.023 & 13.62 & 13.59 & 0.22 & 13.04 & 4.26 \\
\hline Triangle & 0.0025 & 30 & 32.29 & 7.63 & 29.73 & 0.90 \\
\hline Sine & 0.056 & -17.63 & -20.62 & 16.96 & -17.12 & 2.89 \\
\hline Triangle & 0.006 & -12 & -13.92 & 16.00 & -12.51 & 4.25 \\
\hline Sine & 0.0905 & -29.91 & -31.43 & 5.08 & -30.28 & 1.24 \\
\hline Triangle & 0.0095 & -6 & -6.82 & 13.67 & -6.51 & 8.50 \\
\hline Sine & 0.1375 & -7.65 & -10.65 & 39.22 & -8.66 & 13.20 \\
\hline Triangle & 0.013 & 24 & 10.13 & 7.79 & 23.69 & 1.29 \\
\hline Sine & 0.1505 & 19.94 & 15.34 & 23.07 & 18.84 & 5.52 \\
\hline Triangle & 0.0185 & -18 & -20.80 & 15.56 & -16.35 & 9.17 \\
\hline
\end{tabular}


TABLE 3: Identification results at five time points below 5\% noise level.

\begin{tabular}{lccr}
\hline Type & Actual load $(\mathrm{N})$ & Tikhonov regularization & TSVD regularization \\
\hline \multirow{2}{*}{ Relative error (\%) } & Sine loads & 24.99 & 7.56 \\
& Triangle loads & 15.63 & 8.54 \\
\hline \multirow{2}{*}{ Correlation coefficient } & Sine loads & 0.9689 & 0.9984 \\
& Triangle loads & 0.9881 & 0.9967 \\
\hline
\end{tabular}

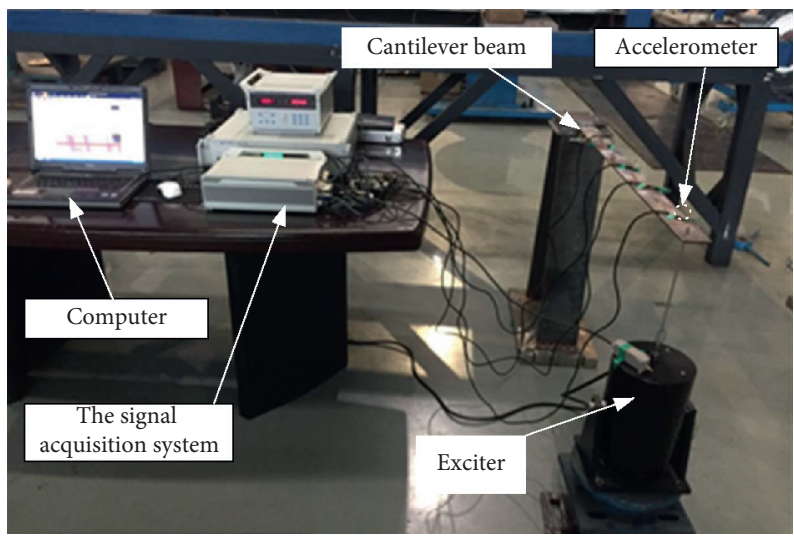

Figure 9: Field diagram of modal experiments of the cantilever beam.

TABLE 4: Natural frequency comparison of beam structures and experimental structures.

\begin{tabular}{lccc}
\hline Modal order & Experimental mode $(\mathrm{Hz})$ & Finite element modal $(\mathrm{Hz})$ & Relative error $(\%)$ \\
\hline 1 & 4.91 & 5.17 & 4.55 \\
2 & 30.64 & 30.79 & -0.49 \\
3 & 85.32 & 76.03 & 7.03 \\
4 & 167.90 & 132.89 & 14.28 \\
5 & 277.21 & 226.95 & 15.49 \\
\hline
\end{tabular}

4.2.2. Model Correction and Sensitivity Analysis. Before performing specific model corrections, it is necessary to determine the correction parameters from the numerous elements. Choosing correction parameters is the key to successful model correction. The interpretability, correctness, and reliability of the correction model greatly depend on the composition of the correction parameters. In addition to the empirical screening method, parameters with higher sensitivity to vibration measurement parameters can also be selected as correction parameters through sensitivity analysis. Sensitivity analysis method is selected to model correction because it can measure the rate of change of structural response caused by the change of parameters or design variables. Through sensitivity analysis, the uncertainty of output variables can be ranked according to the degree of influence of design variables. In order to achieve the correction of the finite element model, the elastic modulus, the linear spring stiffness, and the torsional spring stiffness of the cantilever beam structure are used to design variables.

In order to ensure the accuracy of the dynamic load identification, it is necessary to carefully modify the finite element model in the load identification process. This paper uses several optimization algorithms to modify the finite element model and compares the relative errors of the natural frequency changes after different optimization algorithms modify the model [38].

For the optimization parameters, the equivalent elastic modulus of the beam structure and the stiffness value of the equivalent linear spring are set, and the corresponding upper and lower limits are $1 \times 10^{11} \leq E \leq 3.0 \times 10^{11} \mathrm{MPa}$ and $1 \times 10^{4} \leq K_{1} \leq 1 \times 10^{7} \mathrm{~N} / \mathrm{m}$. The objective function in the optimization process is defined as follows:

$$
\mathbf{f}=\min \left\{\left\|\frac{w_{c}-w_{s}}{w_{s}}\right\|\right\},
$$

where $w_{c}$ represents the calculated natural frequency and $w_{s}$ is the experimentally measured natural frequency.

The target of optimization is the first and second order finite element modal frequencies, and the tolerance of the objective function is set to $10^{-3}$. Set the optimal constraints to ensure that the relative error is less than $5 \%$ for other finite element modal frequencies. In the optimization, the LSGRG (Large Scale Generalized Reduced Gradient), the DS (Downhill Simplex), and the MIGA (Multi-island genetic algorithm) are compared for analysis [39]. The results of comparing the natural frequencies of the 1 st to 5 th modes of the finite element model with the experimental modes are shown in Figure 10. 


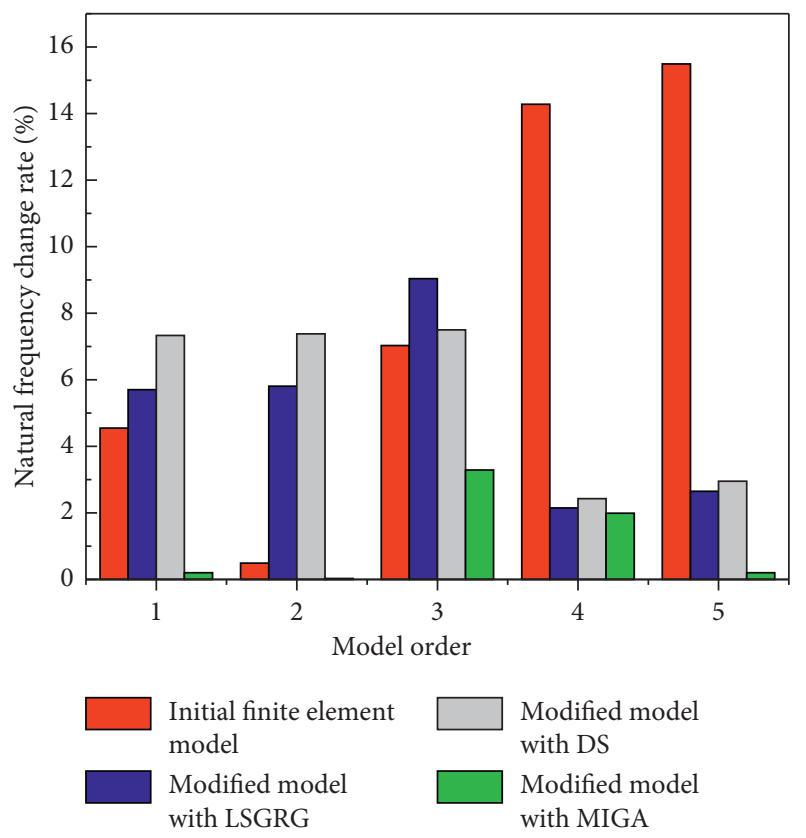

Figure 10: Comparison of natural frequency difference before and after model modification.

From Figure 10, it can be found that the three optimization algorithms LSGRG, DS, and MIGA all have a certain effect on the modified finite element model, especially the correction effect of the MIGA method is the best, and the error rate of the natural frequency is relatively minimal. It shows that the models modified by these three optimization methods can basically meet the requirements of load identification. Of course, in the load identification process, an accurate finite element model is only the basic condition, and the vibration response is also an important indicator to evaluate whether the improved model and the experimental model are suitable. Therefore, the influence of boundary conditions needs to be paid enough attention when carrying out load identification.

4.2.3. Results Analysis and Discussion. In this paper, the multi-island genetic optimization algorithm is mainly used to modify the finite element model in the example, and the accuracy of the TSVD (GCV) regularization method is verified. In order to compare the results of the modified finite element model load identification, an experimental verification analysis is performed. In the test process, the sampling frequency is $2000 \mathrm{~Hz}$ and the sampling time is $2 \mathrm{~s}$. In order to improve the calculation efficiency, load identification is performed on 200 data points among them. Then, the unit pulse loads are applied to the modified model, and the kernel function response is calculated to construct a Green function matrix $G$ for load identification. The loads are identified by the TSVD (GCV) and the Tikhonov (Lcurve) regularization method, respectively. The comparison result is shown in Figure 11.

As can be seen in Figure 11, the experimental results have shown that TSVD has better identification than Tikhonov regularization. According to the comparison of

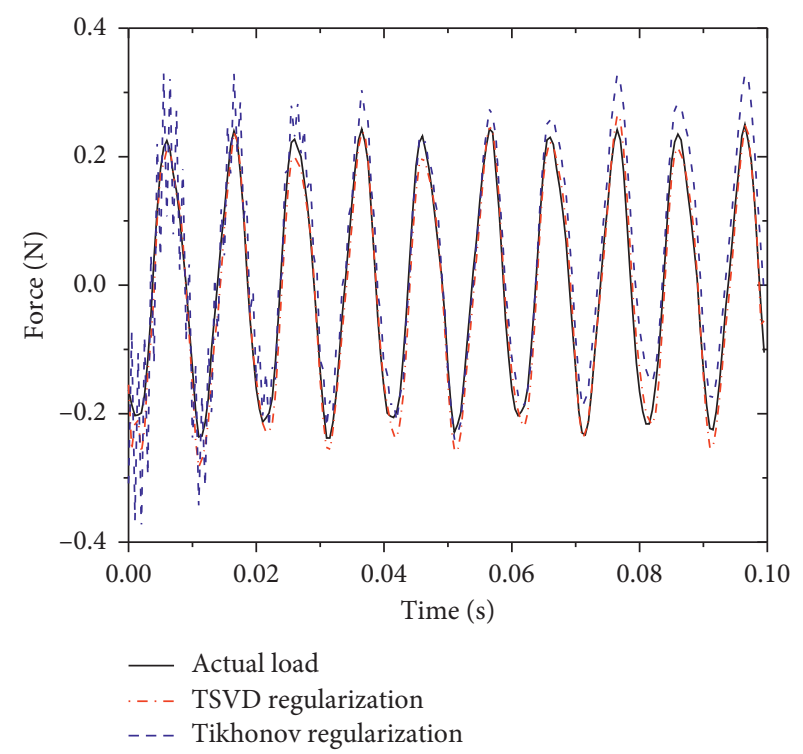

FIgURE 11: The identify results comparison based on different regularization methods.

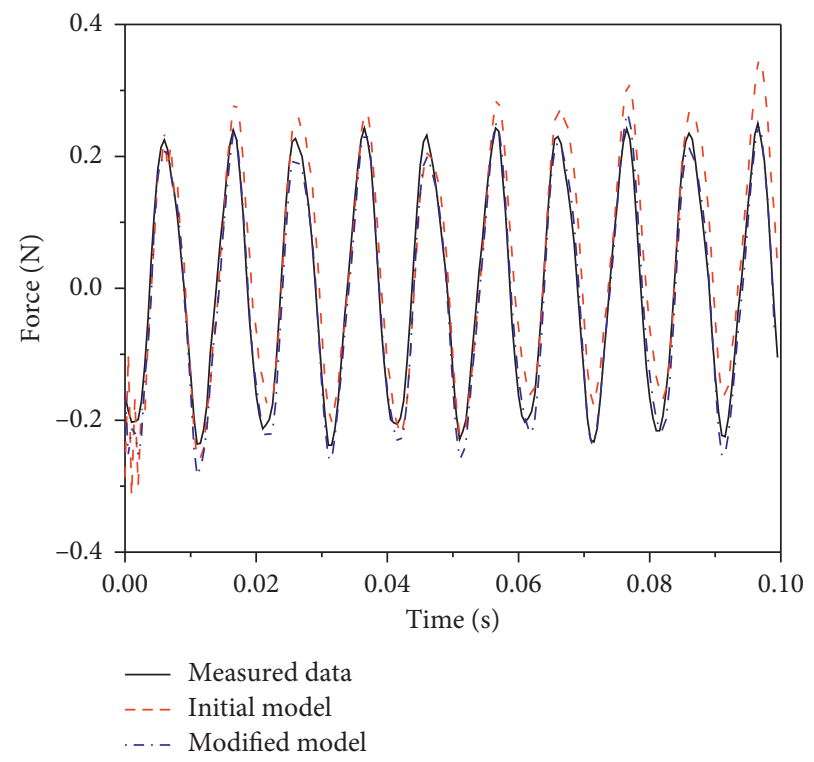

FIgURE 12: Comparison of results after modified finite element model.

simulation and test results, both methods can effectively identify the dynamic load, and the TSVD regularization method has better recognition effect than the Tikhonov method. There is only a slight deviation in the load identification at the peak, which is mainly due to the excessive regularization of the model equation.

In addition, results comparison of the finite element model correction based on the Tikhonov method is shown in Figure 12.

In Figure 12, in order to verify the before and after recognition results of the improved method, the Tikhonov regularization method with better identification effect is 
selected for comparison. The study found that using the load identification method of the modified finite element model, the identification result is closer to the measured load, which is better than the identification effect without the modified model. Through the comparison before and after the recognition results, it is necessary to consider the boundary constraints and the influence of noise. By optimizing the boundary conditions and structural parameters of the simulation model, the recognition effect and accuracy can be further improved.

\section{Conclusion}

In order to improve the accuracy of periodic dynamic load identification in practical engineering, the paper proposes a structural load identification method based on two regularization methods and a finite element correction model through simulation and experimental verification methods. This method compares the results of TSVD and Tikhonov regularization methods and verifies the effectiveness of the method through experiments. The specific conclusions are as follows:

(1) When performing periodic load recognition, although both recognition methods have certain recognition effects, the TSVD method has a better recognition effect on periodic loads and can effectively deal with the ill-conditioned problem of kinematics equations in the load recognition process.

(2) During the test verification process, the improved method can make use of the optimization algorithm to determine the appropriate design variables to ensure that the modified model has a better recognition effect.

(3) When carrying out load identification, the influence of boundary conditions needs to be fully considered. And it is necessary to use optimization algorithms to select design variables to ensure better equivalence of model boundary conditions. At the same time, when performing the finite element model parameter correction based on the actual structural modal measurement results, it is recommended to select the modal with better measured results for correction.

\section{Data Availability}

The data used to support the findings of this study are included within the article. The data are published on figshare website. The files' link can be found in https://figshare.com/ s/79df76d5d71a5606eff8.

\section{Conflicts of Interest}

The authors declare that they have no conflicts of interest.

\section{Acknowledgments}

The authors wish to thank the teachers and students who participated in the research team on Load Identification and
Damage Identification for their contributions. The work was financed by the National Natural Science Foundation of China (51775456) and Self-Developed Research Project of the State Key Laboratory of Traction Power (2019TPL T03).

\section{References}

[1] T. Uhl, "The inverse identification problem and its technical application," Archive of Applied Mechanics, vol. 77, no. 5, pp. 325-337, 2006.

[2] B. Qiao, J. Liu, J. Liu, Z. Yang, and X. Chen, "An enhanced sparse regularization method for impact force identification," Mechanical Systems and Signal Processing, vol. 126, pp. 341367, 2019.

[3] B. Qiao, X. Zhang, J. Gao, R. Liu, and X. Chen, "Sparse deconvolution for the large-scale ill-posed inverse problem of impact force reconstruction," Mechanical Systems and Signal Processing, vol. 83, pp. 93-115, 2017.

[4] A. N. Thite and D. J. Thompson, "The quantification of structure-borne transmission paths by inverse methods. Part 2: use of regularization techniques," Journal of Sound and Vibration, vol. 264, no. 2, pp. 433-451, 2003.

[5] J. Liu, X. Han, C. Jiang, H. M. Ning, and Y. C. Bai, "Dynamic load identification for uncertain structures based on interval analysis and regularization method," International Journal of Computational Methods, vol. 8, no. 4, pp. 667-683, 2011.

[6] K. Liu, S. S. Law, X. Q. Zhu, and Y. Xia, "Explicit form of an implicit method for inverse force identification," Journal of Sound and Vibration, vol. 333, no. 3, pp. 730-744, 2014.

[7] X. Sun, J. Liu, X. Han, C. Jiang, and R. Chen, "A new improved regularization method for dynamic load identification," Inverse Problems in Science and Engineering, vol. 22, no. 7, pp. 1062-1076, 2014.

[8] Y. Jia, Z. Yang, and Q. Song, "Experimental study of random dynamic loads identification based on weighted regularization method," Journal of Sound and Vibration, vol. 342, pp. 113123, 2015.

[9] W. Gao, K. Yu, and Y. Wu, "A new method for optimal regularization parameter determination in the inverse problem of load identification," Shock and Vibration, vol. 2016, pp. 1-16, 2016.

[10] J. Prawin and A. Rama Mohan Rao, "An online input force time history reconstruction algorithm using dynamic principal component analysis," Mechanical Systems and Signal Processing, vol. 99, pp. 516-533, 2018.

[11] Y. M. Mao, X. L. Guo, and Y. Zhao, "A state space force identification method based on Markov parameters precise computation and regularization technique," Journal of Sound and Vibration, vol. 329, no. 15, pp. 3008-3019, 2010.

[12] L. Wang, X. Han, J. Liu, and J. Chen, "An improved iteration regularization method and application to reconstruction of dynamic loads on a plate," Journal of Computational and Applied Mathematics, vol. 235, no. 14, pp. 4083-4094, 2011.

[13] J. Liu, X. Sun, X. Han, C. Jiang, and D. Yu, "A novel computational inverse technique for load identification using the shape function method of moving least square fitting," Computers \& Structures, vol. 144, pp. 127-137, 2014.

[14] J. Liu, X. Meng, C. Jiang, X. Han, and D. Zhang, "Timedomain Galerkin method for dynamic load identification," International Journal for Numerical Methods in Engineering, vol. 105, no. 8, pp. 620-640, 2016.

[15] J. Liu, X. Meng, D. Zhang, C. Jiang, and X. Han, “An efficient method to reduce ill-posedness for structural dynamic load 
identification," Mechanical Systems and Signal Processing, vol. 95, pp. 273-285, 2017.

[16] B. Miao, F. Zhou, C. Jiang, X. Chen, and S. Yang, "A comparative study of regularization method in structure load identification," Shock and Vibration, vol. 2018, pp. 1-8, 2018.

[17] T. Wang, Z. Wan, X. Wang, and Y. Hu, "A novel state space method for force identification based on the Galerkin weak formulation," Computers \& Structures, vol. 157, pp. 132-141, 2015.

[18] T. Lai, T.-H. Yi, H.-N. Li, and X. Fu, "An explicit fourth-order Runge-Kutta method for dynamic force identification," International Journal of Structural Stability and Dynamics, vol. 17, no. 10, 2017.

[19] X. Li, H. Zhao, Z. Chen, Q. Wang, J.-a. Chen, and D. Duan, "Force identification based on a comprehensive approach combining Taylor formula and acceleration transmissibility," Inverse Problems in Science and Engineering, vol. 26, no. 11, pp. 1612-1632, 2018.

[20] J. Sanchez, "Asymptotic approximation method of force reconstruction: application and analysis of stationary random forces," Journal of Sound and Vibration, vol. 424, pp. 318-334, 2018.

[21] Z. Boukria, P. Perrotin, A. Bennani, F. Dupray, and A. Limam, "Structural monitoring: identification and location of an impact on a structurally dissipating rock-shed structure using the inverse method," European Journal of Environmental and Civil Engineering, vol. 16, no. 1, pp. 20-42, 2012.

[22] H. Kalhori, L. Ye, S. Mustapha, J. Li, and B. Li, "Reconstruction and analysis of impact forces on a steel-beamreinforced concrete deck," Experimental Mechanics, vol. 56, no. 9, pp. 1547-1558, 2016.

[23] H. Kalhori, M. M. Alamdari, and L. Ye, "Automated algorithm for impact force identification using cosine similarity searching," Measurement, vol. 122, pp. 648-657, 2018.

[24] J. Sanchez and H. Benaroya, "Review of force reconstruction techniques," Journal of Sound and Vibration, vol. 333, no. 14, pp. 2999-3018, 2014.

[25] D. Calvetti, S. Morigi, L. Reichel, and F. Sgallari, "Tikhonov regularization and the L-curve for large discrete ill-posed problems," Journal of Computational and Applied Mathematics, vol. 123, no. 1-2, pp. 423-446, 2000.

[26] M. Aucejo and O. De Smet, "A multiplicative regularization for force reconstruction," Mechanical Systems and Signal Processing, vol. 85, pp. 730-745, 2017.

[27] B. Qiao, X. Chen, X. Xue, X. Luo, and R. Liu, “The application of cubic B-spline collocation method in impact force identification," Mechanical Systems and Signal Processing, vol. 6465, pp. 413-427, 2015.

[28] B. Qiao, X. Zhang, J. Gao, and X. Chen, "Impact-force sparse reconstruction from highly incomplete and inaccurate measurements," Journal of Sound and Vibration, vol. 376, pp. 72-94, 2016.

[29] P. Ghaderi, A. J. Dick, J. R. Foley, and G. Falbo, "Practical high-fidelity frequency-domain force and location identification," Computers \& Structures, vol. 158, pp. 30-41, 2015.

[30] C. Renzi, C. Pézerat, and J.-L. Guyader, "Vibratory source identification by using the Finite Element Model of a subdomain of a flexural beam," Journal of Sound and Vibration, vol. 332, no. 3, pp. 545-562, 2013.

[31] X. Xue, X. Chen, X. Zhang, and J. Geng, "Load identification in one dimensional structure based on hybrid finite element method," Science China Technological Sciences, vol. 60, no. 4, pp. 538-551, 2016.
[32] A. Esfandiari, F. Bakhtiari-Nejad, M. Sanayei, and A. Rahai, "Structural finite element model updating using transfer function data," Computers \& Structures, vol. 88, no. 1-2, pp. 54-64, 2010.

[33] S. Pradhan and S. V. Modak, "Normal response function method for mass and stiffness matrix updating using complex FRFs," Mechanical Systems and Signal Processing, vol. 32, pp. 232-250, 2012.

[34] D. Shan, Q. Li, I. Khan, and X. Zhou, "A novel finite element model updating method based on substructure and response surface model," Engineering Structures, vol. 103, pp. 147-156, 2015.

[35] B. Gou, W. Zhang, Q. Lu, and B. Wang, "A Successive Selection Method for finite element model updating," $M e$ chanical Systems and Signal Processing, vol. 70-71, pp. 320-333, 2016.

[36] Z. Chen, T. H. T. Chan, A. Nguyen, and L. Yu, "Identification of vehicle axle loads from bridge responses using preconditioned least square QR-factorization algorithm," Mechanical Systems and Signal Processing, vol. 128, pp. 479-496, 2019.

[37] Z. Chen and T. H. T. Chan, "A truncated generalized singular value decomposition algorithm for moving force identification with ill-posed problems," Journal of Sound and Vibration, vol. 401, pp. 297-310, 2017.

[38] J. Sobieszczanski-Sobieski, Multidisciplinary Optimization for Engineering Systems: Achievements and Potential," Optimization: Methods and Applications, Possibilities and Limitations, Springer, Berlin, Germany, 1989.

[39] R. Su, X. Wang, L. Gui, and Z. Fan, "Multi-objective topology and sizing optimization of truss structures based on adaptive multi-island search strategy," Structural and Multidisciplinary Optimization, vol. 43, no. 2, pp. 275-286, 2011. 